\title{
Pharmacotherapy of Insomnia in Children
}

\author{
Rafael Pelayo $^{1} \cdot$ Shehlanoor Huseni $^{1}$
}

Published online: 4 February 2016

(C) Springer International Publishing AG 2016

\begin{abstract}
Insomnia is defined as the inability to fall asleep and/or the inability to maintain sleep to the point that it has a negative impact on time awake. Insomnia in adults may lead to daytime fatigue and sleepiness but in children, the lack of quality sleep may lead to daytime behavioral problems such as hyperactivity and academic difficulty. The pharmacological treatment of insomnia in children is a challenging problem for clinicians since there is paucity of Food and Drug Administration (FDA) approved. Parents often seek medical attention for their children when their own or other members of the family's sleep schedule has been disrupted. The clinician has to take into account the entire family when evaluating a child and making therapeutic recommendations. When evaluating a child with insomnia, it is not only important to take a thorough history of the child's sleep schedule but it also involves getting a detailed evaluation of the other family member's sleep history. The pharmacological management of insomnia is dependent on the child's age. The primary healthcare physician may have limited training in pediatric sleep disorders. Although the mainstay treatment for insomnia in children is behavioral therapy, in this chapter, we will provide a review of the medications that may be used as an adjunct treatment for pediatric insomnia.
\end{abstract}

This article is part of the Topical Collection on Sleep in Children

Rafael Pelayo

pelayo@stanford.edu

1 Sleep Medicine Division, Psychiatry and Behavioral Medicine, Stanford Sleep Disorders Clinic, Stanford University School of Medicine, 450 Broadway Street, Redwood City, CA 94063, USA
Keywords Sleep $\cdot$ Insomnia $\cdot$ Pharmacology . Pharmacotherapy · Children · Adolescents · Infants · Sleep disorders $\cdot$ Antihistamines $\cdot$ Antidepressants $\cdot$ Hypnotics . Benzodiazepines · Trazodone $\cdot$ Doxepin $\cdot$ Clonazepam · Melatonin · Clonidine

\section{Introduction}

Not many things will bring the typical busy sleep medicine practice to a crawl than unexpectedly encountering a parental request for medication for a child with insomnia. Unfortunately, there are no quick answers or established protocols that can be universally applied. Before clinicians can discuss any medication, they have to first take time to understand why the child has insomnia as well as learn what, if any, behavioral and pharmacological interventions have been previously tried. The clinician must take even more time to explain what behavioral interventions may be appropriate for the specific situation. Only after going through this process is it appropriate to proceed with a discussion of pharmacological options for insomnia in a child.

The pharmacological options for treatment of insomnia in children are limited [1]. Often if pharmacological treatment is used, it is usually "off label" because the Food and Drug Administration (FDA) has not approved any prescription hypnotics for people younger than 18 years old. Since behavioral modification has been the mainstay treatment for insomnia in children, pharmacological treatment is generally considered in combination with behavioral therapy. So before we attempt to get our hypothetical clinic moving again by discussing the parent's request for medication for their child's insomnia, we should take a step back and consider what we can learn from our prior collective clinical experience with this situation. 
One of the oldest medications prescribed for sleep in children was chloral hydrate. It is no longer readily available in the USA. Its sedative properties were recognized about 150 years ago. Until fairly recently, it was one of the mainstays of pediatric sedation and insomnia treatment. The author (RP) recalls as a trainee, chloral hydrate was the only option offered to sedate a neurologically impaired child before a procedure, such as a CT scan. One of the attractive features of this medication was that it was delivered in a syrup form making it relatively easy to administer. Many children were able to quickly fall asleep and have successful procedures. However, sometimes children would become extremely agitated when they took the medication. This was described as a "paradoxical reaction" and typically no further explanation would be offered to the bewildered parents. However, patients and their families deserve a better explanation. Why would a sedating medication have such a profoundly opposite effect? In retrospect, these paradoxical reactions may have been due to inadequate dosing, not taking into account the circadian principles of sleep tendencies, or the child's state of mind at the time. In the absence of clear pharmacological guidelines, the natural inclination is to give the lowest possible dose of a medication to a child. However, when treating a child, especially one with a neurological or psychiatric condition, if the dose of medication is underestimated or administered at a time of heighten circadian alertness, the medication may disinhibit the child or create a dissociative state. The child may not have understood they were expected to go to sleep and may have had heightened arousal due to the novel and/or perceived scary environment. The emergence of hypnagogic hallucinations may frighten the child or be misinterpreted as a delirium or psychotic reaction. Prior food intake may also interfere with absorption of the medication, or the child's usual medications may have induced hepatic enzymes or have other interactions further complicating the hypnotic administration consideration [2].

The FDA in 2006 took regulatory action against manufacturers of chloral hydrate. This resulted in discontinuation of its production for the USA. These past experiences should be remembered because they illustrate principles and considerations that must be applied to modern day sedative hypnotics use in children.

It is important to keep the above example in mind, when choosing among current pharmacological options in children with insomnia. We have relatively limited available choices and even fewer choices if we categorically disregard medications that have had prior reports of "paradoxical reactions." It is important to take the time to get a more detailed understanding of circumstance surrounding that putative adverse effect. It is possible that an agent that failed in the past will actually be useful when properly administered. Clinicians must bear in mind that the use of the hypnotics discussed below are not FDA approved for insomnia in children and should inform the family of this. Clinical decisions are therefore carried out on a case-by-case basis (see Table 1).

\section{Age-Related Manifestations of Insomnia}

Insomnia in children is not a homogenous entity, and it is important to briefly review the typical age-related presentations and causes of insomnia. Occult physical reasons for disrupted sleep include allergies, gastroesophageal reflux, and sleep disordered breathing. In infants, insomnia may be related to nocturnal feeding, sleep onset association type, or behavioral insomnia of childhood. Infants learn to associate certain behaviors with sleep onset. Infants will learn associations for sleep. The associations children make with sleep,

Table 1 Selected hypnotics that been used in children

\begin{tabular}{|c|c|c|c|c|}
\hline Drug & Potential application & Dose & Side effects & Formulation \\
\hline Diphenhydramine & Transient insomnia & $0.5 \mathrm{mg} / \mathrm{kg} 25 \mathrm{mg} \max$ & Anticholinergic side effects & $\begin{array}{l}\text { Tablet, capsule, syrup, } \\
\text { injectable }\end{array}$ \\
\hline Melatonin & $\begin{array}{l}\text { Delayed sleep phase syndrome/ } \\
\text { sleep onset insomnia }\end{array}$ & $2.5-10 \mathrm{mg}$ & $\begin{array}{l}\text { Possible exacerbation of } \\
\text { auto-immune disease }\end{array}$ & Tablet \\
\hline Clonazepam $^{\mathrm{a}}$ & $\begin{array}{l}\text { Insomnia associated } \\
\text { with parasomnias }\end{array}$ & $0.5-5 \mathrm{mg}$ & Impairment of respiratory function & Tablet, transdermal patch \\
\hline Zolpidem $^{\mathrm{a}}$ & & $5-10 \mathrm{mg}$ & Next-day sedation & $\begin{array}{l}\text { Tablet, oral spray, } \\
\text { sublingual tablet }\end{array}$ \\
\hline Zaleplon $^{\mathrm{a}}$ & & $5-10 \mathrm{mg}$ & Next-day sedation & Capsules \\
\hline Eszolpiclone $^{\mathrm{a}}$ & & $1-3 \mathrm{mg}$ & Next-day sedation & Tablets \\
\hline Ramelteon $^{\mathrm{a}}$ & & $8 \mathrm{mg}$ & Dizziness/nausea/nipple discharge & Tablets \\
\hline Clonidine & & $0.025-0.3 \mathrm{mg}$ & Bradycardia/hypotension & Tablet/transdermal patch \\
\hline Trazadone & & $20-50 \mathrm{mg}$ & Cardiac arrhythmias & Tablets \\
\hline
\end{tabular}

${ }^{\mathrm{a}}$ FDA approved only in adults 
such as the presence of a parent or feeding with sleep onset, help them to sleep throughout the night. If these associations are not present, the infant may have difficulty in sleeping. Behavioral insomnia can be differentiated from insomnia due to a physical problem by observing how the child sleeps with and without the parent in the same space. If the child sleeps better with the parent, then more likely the child is experiencing behavioral insomnia. If the infant sleeps poorly even with the parent, then a physical problem should be more closely considered.

For otherwise healthy infants, one of the first medications they ever receive is over-the-counter acetaminophen for fever associated with immunizations or viral illness, or teething pain. During these times, the infant may appear to sleep better than usual to some parents. This may explain why parents may give an infant liquid acetaminophen for insomnia. There is no data that has shown acetaminophen in children has a direct effect on sleep. The clinician should specifically ask about this when taking a history since the parents may not volunteer this information. Some parents may also give alcohol to their infants to help them sleep, and this is also important to discuss with the parents. For infants, the hypnotic agents usually recommended by physicians are antihistamines, which are further discussed below. Since they are also available over the counter, parents may not volunteer that they have already tried them.

In older toddlers and young school age children, problems with sleep may be related to nocturnal fears or behavioral insomnia of childhood, limit-setting type. This is an age where children benefit from a predictable soothing bedtime routine and sleep environment, especially if they are sleeping alone. At this age, the child may challenge the parent around bedtime. This challenging behavior may disrupt the pattern of sleep of the household. The child may express fear of the dark that may in reality be a fear of being left alone since they associate darkness with being alone in their bedrooms. Often, behavioral therapy is a sufficient treatment for this insomnia pattern. The principals of cognitive behavior therapy for insomnia in adults can be applied to children. However, in the situation of an extremely anxious or special need child, the parents may request adjunctive pharmacotherapy. Before making any pharmacological recommendations, the clinician should assess for possible undiagnosed obstructive sleep apnea or restless leg syndrome by doing an overnight polysomnography. The medications physicians are likely to recommend in this group are also the antihistamines since they are available in liquid form. Other medications used in this age group are benzodiazepines such as clonazepam that is available in a sublingual form. Melatonin is also often tried by parents and clinicians in this age group [3-6]. Some clinicians, in particular child psychiatrists, will recommend clonidine, an alpha 2 receptor agonist, in this population. The use of a wide range of sedating medications is in clinical use in these children including trazodone, atypical antipsychotics, anticonvulsants, and short-acting hypnotics [7]. If the insomnia persists, as the child grows older, many agents may have been unsuccessfully tried.

In adolescents, the most common form of insomnia is sleep onset in the form of delayed sleep phase disorder. This type of insomnia is categorized as a circadian rhythm disorder. Often, the adolescent prefers to stay up later during the night and has difficulty waking up in the morning to meet societal expectations. Accompanying mood changes may be misdiagnosed as depression [8-10]. Unlike adults with sleep maintenance insomnia, these adolescents typically are able to sleep through the night once they manage to fall asleep. Behavioral therapy for this disorder can include exposure to bright light during desired wake-up time or phototherapy [11-13]. Pharmacotherapy with melatonin can also be used to shift the sleep-wake schedule [14]. Relapse back to the delayed sleep schedule is common when treatment is discontinued. Therefore, the adolescent and family members may need to be informed that this may be a long-term treatment [12].

\section{Pharmacotherapy}

\section{Antihistamines}

Antihistamines are available over the counter and are marketed to treat symptoms of insomnia in adults. One of the more frequently used antihistamines is diphenhydramine, an $\mathrm{H} 1$ receptor antagonist that has effects on both the peripheral and central nervous systems. In the central nervous system, histamine is produced in the posterior hypothalamus. H1 receptor sites are located in the frontal lobe and deep structures of the brain. Histamine is considered a wakefulness promoter as it is involved in arousals, memory, cognition, and regulation of sleep-wake cycles.

Antihistamines are absorbed via the gastrointestinal tract and have a peak blood and tissue level in $2 \mathrm{~h}$. Antihistamines given shortly before bedtime lead to significant decrease in sleep latency time and decrease in the number of awakenings. Centrally acting $\mathrm{H} 1$ antagonists have been found to suppress REM sleep. Duration of treatment with an antihistamine is usually $4-6 \mathrm{~h}$. The half-life of diphenhydramine is shorter in children than it is in adults. The lowest effective dose in children is $0.5 \mathrm{mg} / \mathrm{kg}$ with a maximum dose of $25 \mathrm{mg}$. Adverse effects at therapeutic doses include drowsiness. Overdoses can produce anticholinergic effects that include dilated pupils, flushing, hyperthermia, dry mouth/skin, and agitations/hallucinations (blind as a bat, red as a beet, hot as a hare, dry as a bone, mad as a hatter). Rarely, 
overdoses can produce seizures, catatonic stupor, cardiac rhythm disturbances, and respiratory insufficiency.

\section{Melatonin and Melatonin Agonists}

Melatonin is a hormone that is secreted by the pineal gland. The pineal gland secretes this hormone in a circadian pattern with high plasma levels during the night and low levels during the day, but its release is suppressed by light exposure during the night. One to $2 \mathrm{~h}$ prior to bedtime, plasma melatonin levels usually increase to 10-15-fold higher than daytime levels. Melatonin is at very low levels during the second and third month of life and then it steadily increases. During this time, babies rely on the mothers circadian melatonin levels through breast milk and therefore the babies follow the circadian pattern of the mother.

Melatonin is frequently used for delayed sleep phase syndrome and jet lag as it may have a phase-shifting effect on the suprachiasmatic nucleus. Normal nocturnal plasma ranges in adults are around $50-500 \mathrm{pcg} / \mathrm{ml}$. In adults, taking melatonin in low doses of $0.1-0.3 \mathrm{mg} 2 \mathrm{~h}$ prior to bedtime is thought to be effective in raising the plasma melatonin to normal levels. The doses which may be effective in children are unclear [3, 4, 15-19].

Melatonin is used in children with insomnia due to circadian factors such as sleep phase delay disorder and blindness and patients with midline brain defects such as agenesis of corpus collosum as this may affect the pineal gland. Melatonin has also been used in patients with ADHD and chronic insomnia to reduce sleep onset time. Any data on efficacy in any of these conditions?

Ramelteon, a melatonin receptor agonist, has been approved for use in patients 18 years and older with sleep onset insomnia $[20,21]$. The half-life of this drug is about $1-3 \mathrm{~h}$. Common side effects include headache, somnolence, and fatigue. It may be considered in adolescents with sleep phase delay syndrome. However, no clinical trials are available for patients younger than 18 years old.

Tasimelteon is a melatonin receptor agonist that was released by the FDA for the treatment of non-24-h sleep-wake disorder in 2014. It has an affinity for human melatonin receptors MT1 and MT2, with a higher affinity for the latter. The clinical trials were done in blind adults with a circadian rhythm sleep disorder $[22,23]$. Safety and efficacy have not been established in pediatric patients. There is a hypothetical possibility that this new medication may be useful to help entrain the sleep of children with significant circadian disruption to a more predictable schedule. However, to date, there are no pediatric studies available.

Melatonin can cause enhanced immune function, and therefore it should be used with caution in patients on immunosuppressant medications and/or with immune disorders.
The FDA does not regulate the use of melatonin as it is currently used as a dietary supplement. It should be noted that melatonin and melatonin agonists lack the potential for addiction associated with some other hypnotics.

\section{Clonidine}

Clonidine is an alpha2 receptor agonist. It is commonly used to treat hypertension, but it has also been found to have sedative effects. The exact mechanism of this drug is unknown, but it has been postulated that clonidine acts on the presynaptic terminals of noradrenergic neurons that leads to decrease in norepinephrine release. The onset on this drug is within $1 \mathrm{~h}$ and peak effects are within 2-4 h. It is excreted primarily in urine. Side effects include anticholinergic effects, hypotension, and bradycardia. Clonidine also leads to REM suppression and REM rebound with discontinuation. Clonidine has been widely used in pediatric patients with ADHD and/or neurocognitive impairments and sleep disorders [24]. There is no specified dose approved for hypnotic use, but often, doses of $50 \mathrm{mcg}$ per day is used and gradually increased.

\section{Benzodiazepines}

Benzodiazepines are hypnotics that act as a GABA receptor agonist. The most commonly used benzodiazepine for sleep in children is clonazepam. It is also used for arousal parasomnias such as sleep walking and sleep terrors in children [25]. Clonazepam may allow the child to sleep throughout the night and is thought to decrease the arousal threshold and is usually prescribed at a dose of $0.25-0.5 \mathrm{mg}$. It is available in quick dissolving wafer formulation for children that cannot swallow pills. Benzodiazepines may lead to muscle relaxation, and therefore caution is advised in children with obstructive sleep apnea as it may exacerbate the condition.

\section{Selective Benzodiazepine Receptor Agonist (AKA Non-Benzodiazepine Hypnotics)}

Selective benzodiazepine receptor agonist including zolpidem, eszolpiclone, and zaleplon are thought to offer some advantages compared to nonselective benzodiazepines; at low dosages, they are thought to preserve the overall sleep architecture and may have less insomnia rebound effects after discontinuation when compared to nonselective benzodiazepines. These medications only have FDA approval for use in patients older than 18 years old, and therefore, the use of these medications in children is considered "off-label." They may also be difficult to administer in children as none of these medications are offered in liquid or dissolvable wafer form [26]. There are reports of zolpidem abuse among adolescents $[27,28]$. 
Physicians should take into account the half-life of the selective benzodiazepine receptor agonist as it may help them to decide if they want to use it to shorten sleep latency or to increase total sleep time. Zaleplon has a half-life of only $1 \mathrm{~h}$ and therefore may be a reasonable choice to use in adolescents with delayed sleep phase disorder as it will shorten sleep latency and will be eliminated well before wake time. It may also lead to decreased side effect of next day sedation. Zolpidem's half-life is slightly higher at $2.5 \mathrm{~h}$ and eszopiclone has a half-life of $6 \mathrm{~h}$.

\section{Hypocretin/Orexin Receptor Antagonist}

Suvorexant is the first hypocretin/orexin receptor antagonist to be approved for the treatment of insomnia in adults [29, 30]. Hypocretin/orexin is a neuropeptide that regulates arousal and wakefulness. Mutations and deficits in the hypocretin/orexin system are linked with narcolepsy. The clinical trials in adults found the medication to be effective for insomnia in adults [31]. No studies are available on the safety and efficacy in children.

\section{Antidepressants}

Antidepressant medications can have sedating properties and have been used for sleep in children in an off-label fashion. Perhaps the most commonly used antidepressant for sleep is trazodone [7]. In the author's experience (RP), this is not an ideal choice due to unproven efficacy and potential side effects such as anticholinergic effects. An antidepressant approved for adults is doxepin at a low dose of 3 or $6 \mathrm{mg}$ for sleep maintenance insomnia (as opposed to sleep onset) [32]. The author prescribed it to one child with autistic behavior who had disruptive nighttime awakenings but did not have difficulty falling asleep. The family expressed gratitude at the improved sleep. There are no clinical trials in children.

Also, can you add something about the use of atypical antipsychotics? These are also used in children, particularly those with developmental delays.

\section{Conclusion}

Pharmacological treatment of insomnia in children should typically be combined with behavioral therapy. Most pharmacological treatments for insomnia are not FDA approved and therefore are used "off-label" by many clinicians. Therefore, clinicians should use these medications with caution and with the parents' knowledge of the situation. In considering pharmacological treatment for insomnia, the clinician should assess each patient on a case-to-case basis and consider benefits versus risks of treatment.

\section{Compliance with Ethical Standards}

Conflict of Interest Rafael Pelayo and Shehlanoor Huseni declare that they have no conflict of interest.

Human and Animal Rights and Informed Consent This article does not contain any studies with human or animal subjects performed by any of the authors.

\section{References}

1. Troester MM, Pelayo R. Pediatric sleep pharmacology: a primer. Semin Pediatr Neurol. 2015;22(2):135-47.

2. Pelayo R, Yuen K. Pediatric sleep pharmacology. Child Adolesc Psychiatr Clin N Am. 2012;21(4):861-83.

3. Bruni O, Alonso-Alconada D, Besag F, Biran V, Braam W, Cortese $\mathrm{S}$, et al. Current role of melatonin in pediatric neurology: clinical recommendations. Eur J Paediatr Neurol. 2015;19(2):122-33.

4. Melatonin for sleep problems in children with neurodevelopmental disorders. Drug Ther Bull. 2015;53(10):117-20.

5. Malow B, Adkins KW, McGrew SG, Wang L, Goldman SE, Fawkes D, et al. Melatonin for sleep in children with autism: a controlled trial examining dose, tolerability, and outcomes. J Autism Dev Disord. 2012;42(8):1729-37. author reply 38.

6. van Geijlswijk IM, van der Heijden KB, Egberts AC, Korzilius HP, Smits MG. Dose finding of melatonin for chronic idiopathic childhood sleep onset insomnia: an RCT. Psychopharmacology (Berlin). 2010;212(3):379-91.

7. Owens JA, Rosen CL, Mindell JA, Kirchner HL. Use of pharmacotherapy for insomnia in child psychiatry practice: a national survey. Sleep Med. 2010;11(7):692-700.

8. Sivertsen B, Harvey AG, Pallesen S, Hysing M. Mental health problems in adolescents with delayed sleep phase: results from a large population-based study in Norway. J Sleep Res. 2015;24(1):11-8.

9. Auger RR, Burgess HJ, Emens JS, Deriy LV, Thomas SM, Sharkey $\mathrm{KM}$. Clinical practice guideline for the treatment of intrinsic circadian rhythm sleep-wake disorders: advanced sleep-wake phase disorder (ASWPD), delayed sleep-wake phase disorder (DSWPD), non-24-hour sleep-wake rhythm disorder (N24SWD), and irregular sleep-wake rhythm disorder (ISWRD). An update for 2015. J Clin Sleep Med. 2015. doi:10.5664/jcsm.5100.

10. Sivertsen B, Pallesen S, Stormark KM, Boe T, Lundervold AJ, Hysing M. Delayed sleep phase syndrome in adolescents: prevalence and correlates in a large population based study. BMC Public Health. 2013;13:1163.

11. Danielsson K, Jansson-Frojmark M, Broman JE, Markstrom A. Cognitive behavioral therapy as an adjunct treatment to light therapy for delayed sleep phase disorder in young adults: a randomized controlled feasibility study. Behav Sleep Med. 2015;5:1-21.

12. Saxvig IW, Wilhelmsen-Langeland A, Pallesen S, Vedaa O, Nordhus IH, Bjorvatn B. A randomized controlled trial with bright light and melatonin for delayed sleep phase disorder: effects on subjective and objective sleep. Chronobiol Int. 2014;31(1):72-86.

13. Wilhelmsen-Langeland A, Saxvig IW, Pallesen S, Nordhus IH, Vedaa $\mathrm{O}$, Lundervold AJ, et al. A randomized controlled trial with bright light and melatonin for the treatment of delayed sleep phase disorder: effects on subjective and objective sleepiness and cognitive function. J Biol Rhythm. 2013;28(5):306-21.

14. van Geijlswijk IM, Korzilius HP, Smits MG. The use of exogenous melatonin in delayed sleep phase disorder: a meta-analysis. Sleep. 2010;33(12):1605-14.

15. Schwichtenberg AJ, Malow BA. Melatonin treatment in children with developmental disabilities. Sleep Med Clin. 2015;10(2):181-7. 
16. Hartz I, Handal M, Tverdal A, Skurtveit S. Paediatric off-label use of melatonin - a register linkage study between the Norwegian prescription database and patient register. Basic Clin Pharmacol Toxicol. 2015;117(4):267-73.

17. Ayyash HF, Preece P, Morton R, Cortese S. Melatonin for sleep disturbance in children with neurodevelopmental disorders: prospective observational naturalistic study. Expert Rev Neurother. 2015;15(6):711-7.

18. Cummings C, Canadian Pediatric Society CPC. Melatonin for the management of sleep disorders in children and adolescents. Paediatr Child Health. 2012;17(6):331-6.

19. Gitto E, Aversa S, Reiter RJ, Barberi I, Pellegrino S. Update on the use of melatonin in pediatrics. J Pineal Res. 2011;50(1):21-8.

20. Kuriyama A, Honda M, Hayashino Y. Ramelteon for the treatment of insomnia in adults: a systematic review and meta-analysis. Sleep Med. 2014;15(4):385-92.

21. Liu J, Wang LN. Ramelteon in the treatment of chronic insomnia: systematic review and meta-analysis. Int J Clin Pract. 2012;66(9): 867-73.

22. Lockley SW, Dressman MA, Licamele L, Xiao C, Fisher DM, Flynn-Evans EE, et al. Tasimelteon for non-24-hour sleep-wake disorder in totally blind people (SET and RESET): two multicentre, randomised, double-masked, placebo-controlled phase 3 trials. Lancet. 2015;386(10005): 1754-64.

23. Lavedan C, Forsberg M, Gentile AJ. Tasimelteon: a selective and unique receptor binding profile. Neuropharmacology. 2015;91:142-7.
24. Schnoes CJ, Kuhn BR, Workman EF, Ellis CR. Pediatric prescribing practices for clonidine and other pharmacologic agents for children with sleep disturbance. Clin Pediatr (Phila). 2006;45(3):229-38.

25. Kotagal S. Treatment of dyssomnias and parasomnias in childhood. Curr Treat Options Neurol. 2012;14(6):630-49.

26. Blumer JL, Reed MD, Steinberg F, O'Riordan MA, Rosen CL, Springer MA, et al. Potential pharmacokinetic basis for zolpidem dosing in children with sleep difficulties. Clin Pharmacol Ther. 2008;83(4):551-8.

27. Schepis TS. Age cohort differences in the nonmedical use of prescription zolpidem: findings from a nationally representative sample. Addict Behav. 2014;39(9):1311-7.

28. Ford JA, McCutcheon J. The misuse of ambien among adolescents: prevalence and correlates in a national sample. Addict Behav. 2012;37(12):1389-94.

29. Rhyne DN, Anderson SL. Suvorexant in insomnia: efficacy, safety and place in therapy. Ther Adv Drug Saf. 2015;6(5):189-95.

30. Patel KV, Aspesi AV, Evoy KE. Suvorexant: a dual orexin receptor antagonist for the treatment of sleep onset and sleep maintenance insomnia. Ann Pharmacother. 2015;49(4):477-83.

31. Kishi T, Matsunaga S, Iwata N. Suvorexant for primary insomnia: a systematic review and meta-analysis of randomized placebocontrolled trials. PLoS One. 2015;10(8):e0136910.

32. Yeung WF, Chung KF, Yung KP, Ng TH. Doxepin for insomnia: a systematic review of randomized placebo-controlled trials. Sleep Med Rev. 2015;19:75-83. 